\title{
Position statement by Experts of the Polish Menopause and Andropause Society, and the Polish Society of Aesthetic and Reconstructive Gynaecology on the medicinal product Intrarosa ${ }^{\circledR}$
}

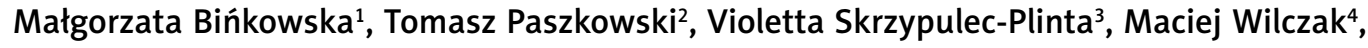 \\ Wojciech Zgliczyński ${ }^{5}$

\begin{abstract}
II Department of Obstetrics and Gynecology, Centre of Postgraduate Medical Education in Warsaw, Poland ${ }^{2}$ III Chair and Department of Gynecology, Medical University of Lublin, Poland ${ }^{3}$ Chair of Women's Health, Medical University of Silesia in Katowice, Poland ${ }^{4}$ Chair and Department of Mother and Child Health, Poznan University of Medical Sciences, Poznan, Poland ${ }^{5}$ Department of Endocrinology, Centre of Postgraduate Medical Education in Warsaw, Poland
\end{abstract}

\section{Introduction}

A set of menopause-related symptoms affecting the external genitalia and lower urinary tract is discussed in contemporary scientific literature under the name of urogenital atrophy (UGA) or vulvovaginal atrophy (VVA) [1]. In 2014, a team of experts from two scientific associations - The North American Menopause Society (NAMS) and The International Society for the Study of Women's Sexual Health (ISSWSH) - proposed a new terminology for postmenopausal atrophic symptoms, replacing the terms VVA and UGA with the term genitourinary syndrome of menopause (GSM) [2]. The change was motivated by the belief that this set of symptoms requires a more holistic therapeutic approach falling outside the scope of interest of gynaecologists and urologists only. The signs and symptoms of GSM are summarised in the Table 1.

The diagnosis of GSM is based primarily on the patient's medical history and the findings of physical examinations, of which the most important one is the speculum examination. Additional examinations confirming the diagnosis of GSM include:

- vaginal $\mathrm{pH}>5$,

- an increase in the number of parabasal cells,

- a decrease in the number of superficial cells in maturation index (MI).

A common feature shared by the symptoms which make up the syndrome is their causal relationship with age-related reductions in both peripheral and local concentrations of sex steroids, particularly oestrogens and androgens [3-5].

Androgens and oestrogens produce both separate and synergistic effects on vaginal morphology and function. The most important types of vaginal effects produced by oestrogens involve vaginal elasticity and $\mathrm{pH}$ level. The impact of androgens is related predominantly to such aspects of vaginal physiology as innervation and contractility of vaginal walls. The common androgenic and oestrogenic area of activity relates to

Table 1. Genitourinary syndrome of menopause (GSM) symptoms and signs

\begin{tabular}{l}
\hline Symptoms \\
\hline Vaginal/vulvar dryness \\
\hline Inadequate lubrication during sexual activity \\
\hline Discomfort or pain during sexual activity \\
\hline Postcoital bleeding \\
\hline Decreased sexual desire, impaired arousal and orgasm \\
\hline Vaginal or vulvar irritation/burning/itching \\
\hline Dysuric symptoms \\
\hline Frequent urination/urinary urgency \\
\hline Signs \\
\hline Decreased vaginal lubrication \\
\hline Decreased vaginal elasticity \\
\hline Resorption of labia minora \\
\hline Vaginal erythema/pallor \\
\hline Loss of vaginal rugae \\
\hline Tissue fragility/tearing/fissures \\
\hline Urethral eversion or prolapse \\
\hline Loss of hymenal remnants \\
\hline Prominence of urethral meatus \\
\hline Introital stenosis/retraction \\
\hline Recurrent urinary tract infections \\
\hline
\end{tabular}


the structure and function of the lamina propria and muscular layer of vaginal walls, as well as its vascular perfusion $[3,4,6]$.

The wide spectrum of GSM-associated symptoms is attributable to a particularly high density of oestrogen and androgen receptors within the external genitalia and the lower urinary tract. Changes caused by hormonal deficiencies are accompanied by a decrease in the tissue content of collagen and elastin, epithelial thinning due to the loss of the superficial layer, dysfunction of smooth muscle cells, and an increase in connective tissue density. Consequences of the changes listed above include greater susceptibility to injuries and discomfort during sexual intercourse, and an altered composition of vaginal microflora manifesting primarily as Lactobacillus deficiency. The aetiopathogenetic chain described above results in a set of symptoms experienced by about $50 \%$ of women in the peri- and postmenopausal periods, which shows that between 2 and 3 million Polish women are affected by this problem [2, 5].

The prevalence of symptoms of vaginal dryness rises in relation to the menopausal status - in the early perimenopausal period the mean prevalence is $4 \%$, growing to $25 \%$ a year after the menopause, and reaching $47 \%$ three years postmenopausally. Approximately $64 \%$ of postmenopausal women experience dyspareunia associated with a decrease in libido leading to the avoidance of sexual activity. In addition, postmenopausal atrophic changes induce urinary tract symptoms such as urinary incontinence (15-35\%), recurrent urinary infections (17\%), and asymptomatic bacteriuria (20\%) [5, 7].

Compared to GSM-free women, patients with symptoms of genitourinary syndrome of menopause have a significantly impaired quality of life $(\mathrm{Q} o \mathrm{~L})$ in a number of aspects including mobility, emotional well-being, sexual functioning and self-concept, and body image. The overall degree of QoL impairment caused by symptoms of GSM is comparable to the impact on the QoL exerted by chronic diseases such as arthritis, chronic obstructive pulmonary disease, asthma, or irritable bowel syndrome [5, 7].

Despite very high prevalence of symptoms of postmenopausal urogenital atrophy, and their devastating effect on the quality of life, the problem surprisingly rarely becomes the subject of medical intervention. This can be attributed to a number of factors. Firstly, only $4 \%$ of patients know that their symptoms are treatable, and only 1 in 4 women experiencing them reports the problem to her doctor. Secondly, only $13 \%$ of doctors discuss the topic of urogenital atrophy with their postmenopausal patients [5, 7].

In many cases, GSM treatment based solely on the topical administration of oestrogens or moisturisers/lubricants fails to provide satisfactory results. Over $65 \%$ of women are dissatisfied with their topical GSM thera- py [8]. For many years, the search has been on for new treatment modalities for GSM, focusing both on the therapeutic efficacy and safety profile. In this context, a significant breakthrough came with the marketing of the first intravaginal drug containing dehydroepiandrosterone (DHEA, prasterone) - the medicinal product Intrarosa ${ }^{\circledR}$. The main distinctive feature of the use of prasterone in topical GSM therapy is the intracrine action of DHEA.

Unlike oestrogens, which are secreted into the blood as active hormones, endogenous DHEA is produced by the adrenal glands (as well as gonads and the brain) and released as an inactive precursor. Inactive DHEA is converted intracellularly to active hormones which produce cellular-specific effects. The amount of active hormones produced in a cell corresponds to the specific needs of that cell. The endocrine mechanism of action of oestrogens and androgens lies in their systemic effect, and in the fact that sex hormones have direct access to receptors, which control local action with no cellular control of the amount of active sex steroid reaching its receptors. In the intracrine mechanism of action, each cell controls its exposure to oestrogens and androgens through more than 30 enzymes that catalyse the synthesis and inactivation of oestrogens and androgens to an extent depending on the needs of the cell. Where DHEA is used for therapy, peripheral cells monitor the exposure to active oestrogens and androgens. As a result of the intracrine action of DHEA, a very small (clinically insignificant) amount of sex hormones diffuses into blood, hence no systemic effects on other tissues are induced by active hormones [9].

\section{Information about Intrarosa $®$ included in the summary of product characteristics [10]}

Intraros $\mathrm{a}^{\circledR}$ is a medicinal product in the form of vaginal pessaries. The active substance contained in the medicinal product is prasterone. Each pessary contains $6.5 \mathrm{mg}$ of prasterone. Intrarosa ${ }^{\circledR}$ is indicated for the treatment of vulvar and vaginal atrophy in postmenopausal women having moderate to severe symptoms. The recommended dose is $6.5 \mathrm{mg}$ prasterone (one pessary) administered once daily, at bedtime.

\section{Pharmacodynamic and pharmacokinetic properties}

Intrarosa ${ }^{\circledR}$ contains prasterone, i.e. dehydroepiandrosterone (DHEA), which is both biochemically and biologically identical to the endogenous human DHEA, a precursor to steroid hormones which is inactive by itself, and converted into oestrogens and androgens. Intravaginally administered prasterone is an inactive precursor which enters the vaginal cells, where it is converted into cell-specific small amounts of both oes- 
trogens and androgens depending upon the activity of enzymes in each cell type. The beneficial effects on the symptoms and signs of vulvar and vaginal atrophy are achieved through the activation of the vaginal oestrogen and androgen receptors.

In a study conducted in postmenopausal women, treatment with Intrarosa ${ }^{\circledR}$ pessaries administered once daily for 7 days resulted in the mean prasterone $C_{\max }$ and mean area under the curve from 0 to 24 hours (AUCO-24) of $4.4 \mathrm{ng} / \mathrm{ml}$ and $56.2 \mathrm{ng} \mathrm{h} / \mathrm{ml}$ at day 7 , respectively. The values were significantly higher than those in the group treated with placebo. The $C_{\max }$ and AUCO-24 values of the testosterone and oestradiol metabolites were also slightly higher in women treated with Intrarosa ${ }^{\circledR}$ pessaries compared to those receiving placebo, but they remained within normal values for postmenopausal women (< $10 \mathrm{pg}$ oestradiol/ml; $<0.26 \mathrm{ng}$ testosterone $/ \mathrm{ml}$ ), as determined by measurements using validated mass spectrometry-based assays both for the study samples and reference values.

\section{Contraindications}

Contraindications to using the medicinal product Intrarosa ${ }^{\circledR}$ include:

- hypersensitivity to the active substance or to any of the excipients,

- undiagnosed genital bleeding,

- diagnosed, suspected or past breast cancer,

- diagnosed or suspected oestrogen-dependent malignant tumours (e.g. endometrial cancer),

- untreated endometrial hyperplasia,
- acute liver disease, or history of liver disease for as long as the results of liver function tests have failed to return to normal,

- previously or currently diagnosed venous thromboembolism (deep vein thrombosis, pulmonary embolism),

- known susceptibility to venous thrombosis (e.g. deficiency of protein C, protein S or antithrombin),

- active or recent arterial thromboembolic disease (e.g. angina, myocardial infarction),

- porphyria.

Before initiating treatment with Intrarosa ${ }^{\circledR}$ a complete personal and family medical history should be taken. A physical evaluation (including gynaecological and breast examination) should be performed, guided by the information obtained from the patient's history and contraindications, and taking into account special warnings and precautions for using the product.

Adverse effects reported during clinical studies investigating the medicinal product Intrarosa ${ }^{\circledR}$ were mild and infrequent. They included an increase in vaginal discharge (5.7-14.2\%), and changes in exfoliative cytology, mainly of the ASCUS type ( $2.1 \%$ of patients).

\section{Evaluation of the efficacy and safety of Intrarosa ${ }^{\oplus}$ based on liferature review}

The efficacy and safety of Intraros ${ }^{\circledR}$ were evaluated in 6 clinical trials involving a total of 1,571 women treated with intravaginal prasterone (Table 2). The studies were conducted in accordance with the Food and Drug Administration (FDA) guidelines for testing drugs indicated for the treatment of menopause-related vag-

Table 2. Characteristics of clinical studies investigating intravaginal use of prasterone

\begin{tabular}{|c|c|c|c|c|c|}
\hline Study code & Study type & Phase & Duration & $\begin{array}{l}\text { Placebo - number } \\
\text { of subjects }\end{array}$ & $\begin{array}{c}\text { Prasterone doses - number } \\
\text { of subjects }\end{array}$ \\
\hline ERC-213 & $\begin{array}{l}\text { Pharmacokinetics; } \\
\text { RCT, double-blind, } \\
\text { single-centre }\end{array}$ & $1-2$ & 1 week & 10 & $\begin{array}{l}6.5 \text { mg: } 10 \\
13 \text { mg: } 10 \\
23.4 \text { mg: } 10\end{array}$ \\
\hline ERC-210 & $\begin{array}{c}\text { Efficacy; } \\
\text { RCT, double-blind, } \\
\text { multicentre }\end{array}$ & 3 & 12 weeks & 53 & $\begin{array}{l}3.25 \text { mg: } 53 \\
6.5 \text { mg: } 56 \\
13 \text { mg: } 54\end{array}$ \\
\hline ERC-231 & $\begin{array}{c}\text { Efficacy; } \\
\text { RCT, double-blind, } \\
\text { multicentre }\end{array}$ & 3 & 12 weeks & 81 & $\begin{array}{l}3.25 \mathrm{mg}: 87 \\
6.5 \mathrm{mg}: 87\end{array}$ \\
\hline ERC-234 & $\begin{array}{c}\text { Efficacy; } \\
\text { RCT, double-blind, } \\
\text { multi-centre }\end{array}$ & 3 & 12 weeks & 152 & $\begin{array}{l}3.25 \mathrm{mg}: 148 \\
6.5 \mathrm{mg}: 150\end{array}$ \\
\hline ERC-230 & $\begin{array}{c}\text { Safety; } \\
\text { open-label, multicentre }\end{array}$ & 3 & 52 weeks & 0 & 6.5 mg: 530 \\
\hline ERC-238 & $\begin{array}{c}\text { Efficacy; } \\
\text { RCT, double-blind, } \\
\text { multi-centre }\end{array}$ & 3 & 12 weeks & 182 & 6.5 mg: 376 \\
\hline Total & & & & 478 & 1,571 \\
\hline
\end{tabular}

RCT - randomized controlled trial 
inal/vulvar atrophy [11], and the study findings were published in peer-reviewed journals.

The efficacy of the approved dose of intravaginal prasterone (6.5 $\mathrm{mg}$ once daily) in postmenopausal women with GSM symptoms was initially determined in the ERC-210 study which compared different doses of prasterone (3.25 mg, $6.5 \mathrm{mg}$ and $13 \mathrm{mg}$ ) vs. placebo [12], and then confirmed in 2 larger-scale phase III, randomised, controlled clinical studies with a duration of 12 weeks (ERC-231 [13] and ERC-238 [14]), and in the 52-week open-label study ERC-230 [15], which was originally planned to evaluate the safety of using prasterone. The studies involved postmenopausal women aged 40-80, with normal mammogram and Pap smear results, without a history of hysterectomy (ERC-230) or regardless of whether hysterectomy was done or not (ERC-231, ERC-238). Women with previous diagnosis of cancer, thromboembolic disease and other gynaecological diseases were excluded from study participation.

\section{Results of clinical trials with a 12 -week time horizon}

The ERC-231 and ERC-238 studies involved women with a baseline vaginal $\mathrm{pH}$ value of $>5$, having $\leq 5 \%$ of superficial cells on vaginal smear, who identified moderate to severe dyspareunia as the most bothersome symptom associated with GSM. In addition to moderate/severe dyspareunia, $81 \%$ and $84 \%$ of women in the ERC-231 and ERC-238 studies, respectively, had moderate to severe vaginal dryness, and $20 \%$ and $39 \%$, respectively, had vaginal itching/irritation $[13,14]$.

The primary endpoints in the ERC-231 and ERC238 studies were changes from the baseline observed after 12 weeks in the proportions of parabasal and superficial cells on vaginal smear, vaginal $\mathrm{pH}$, and an increase in the severity of dyspareunia. If the change in score for dyspareunia vs. placebo was statistically significant, other clinical symptoms of GSM - such as vaginal dryness, vaginal irritation/itching (secondary endpoints) - were assessed. Other secondary endpoints included vaginal discharge, and vaginal epithelial integrity, thickness and colour evaluated on a scale of 0 (no atrophy) to 4 (severe atrophy) based on the physical examination.

Compared with placebo, a 12-week intravaginal prasterone therapy resulted in a significant improvement in all primary endpoints of the study, i.e. a reduction in the proportion of parabasal cells (ERC-231: $-45.8 \%$, ERC-238: $-29.5 \%)$, an increase in the proportion of superficial cells (ERC-231: $+4.7 \%$, ERC-238: $+8.5 \%$ ), a decrease in vaginal pH (ERC-231: -0.8 , ERC-238: $-0.7 \mathrm{pH}$ units), a decreased score for the severity of dyspareunia (ERC-231: -0.4, ERC-238: -0.4) $[13,14]$. Similar results were obtained after pooling data from the prasterone $6.5 \mathrm{mg}$ and placebo arms in the ERC-231, ERC-238 and ERC-210 studies (only the subpopulation in which dyspareunia was considered as the most bothersome symptom of GSM) [16].

Also, a significant improvement in secondary endpoints (vaginal dryness, vaginal discharge, and vaginal epithelial integrity, thickness and colour) was noted in prasterone-treated patients compared to the placebo group.

\section{Long-term efficacy of intravaginal prasterone therapy}

The clinical benefits of prasterone, which were demonstrated in 12-week phase III randomised, placebo-controlled studies, were then confirmed by the ERC-230 study with a 52-week time horizon, in which the primary endpoints were safety parameters, and the secondary endpoints - drug efficacy characteristics [15]. All the women who received prasterone were included in safety analysis $(n=454)$, while the subgroup of patients who considered dyspareunia, vaginal dryness or vaginal irritation/itching as the most bothersome symptom was analysed as a group with menopausal atrophy $(n=292)$. In both populations, after 52 weeks of use, a significant improvement was seen compared to the baseline values in efficacy parameters such as a reduction in the percentage of parabasal cells, an increase in the percentage of superficial cells, and a decrease in vaginal $\mathrm{pH}$, as well as the abundance of vaginal discharge, and vaginal epithelial integrity, thickness and colour. In women who regarded moderate to severe dyspareunia as the most bothersome symptom of GSM, and who met the criteria of postmenopausal vaginal atrophy ( $\leq 5 \%$ of superficial cells on vaginal smear, vaginal $\mathrm{pH}>5)(n=183)$, the pain severity score was reduced by $46.7 \%$ during a period of 12 weeks, and further by $19.4 \%$ between 12 and 52 weeks, reaching $33.9 \%$ of the baseline value at week $52(2.57 \pm 0.04$ at baseline vs. $0.87 \pm 0.07$ at week $52 ; p<0.0001)$. In women with moderate to severe dyspareunia who considered vaginal dryness or vaginal irritation/itching as the most bothersome symptom of atrophy $(n=57)$, the pain severity score decreased from $2.42 \pm 0.07$ at baseline to $0.77 \pm 0.12$ at week 52 $(p<0.0001)$. Also in women with moderate to severe vaginal dryness $(n=251)$ or vaginal irritation/itching $(n=86)$, the severity of each of these symptoms after 52 weeks of using prasterone also decreased significantly $(-1.5$ to $-1.7 ; p<0.001)$. These data indicate that prasterone has a beneficial effect on the reduction of the three manifestations of GSM regardless of whether they are considered the most bothersome symptom or not [15]. 


\section{Other study results}

The effect of intravaginal prasterone therapy on the quality of sexual life in postmenopausal women was assessed using the Female Sexual Function Index (FSFI) questionnaire in 2 clinical studies: ERC-238 [17] and ERC-239 [18]. In the ERC-238 study, prasterone used for 12 weeks in postmenopausal women with moderate to severe dyspareunia symptoms caused a statistically significant improvement compared to the placebo in each domain of the FSFI questionnaire (from $+33 \%$ to $+56.8 \%)$, and in the total FSFI score $(+41.3 \%$ vs. placebo). Treatment with prasterone was also found to have a positive effect on the opinions of sexual partners of the study women: $36 \%$ of men with a prasterone-treated partner did not experience vaginal dryness at the end of therapy, compared to $7.8 \%$ in the placebo group [19]. A positive effect of prasterone on the quality of sexual life was also demonstrated in the ERC-230 study in which a total of 154 women completed the FSFI questionnaire. After 52 weeks, there was a statistically significant improvement compared to the baseline scores in each domain of the questionnaire separately and in the total FSFI score [18].

\section{Tolerability profile and safety of intravaginal prasterone therapy}

Based on the pooled results of 6 phase I-III studies (including ERC-210, ERC-230, ERC-231, and ERC-238), the safety profile of prasterone was similar to placebo over a time horizon of up to 16 weeks [16]. The prevalence of adverse events in women receiving $6.5 \mathrm{mg}$ of prasterone/day $(n=1,196)$ was $52.4 \%$ vs. $47.7 \%$ in the placebo group $(n=474)$. The most common adverse reaction occurring in $>5 \%$ of women, with a higher frequency in the prasterone group than in the placebo group, was increased vaginal discharge (8.3\% vs. $3.4 \%)$. Less than $1 \%$ of women taking prasterone at doses of 3.25-13 mg discontinued the drug because of adverse reactions. Other adverse reactions which are more frequent in the prasterone group than in the placebo group include urinary tract infections ( $4.8 \% \mathrm{vs}$. $4.4 \%$ ), weight fluctuations (weight increase in $2.5 \%$ vs. $1.3 \%$, weight reduction in $2.6 \%$ vs. $1.3 \%$ of women), cervical dysplasia (1.8\% vs. $1.3 \%)$, and sinusitis (1.6\% vs. $1.5 \%)$ [16].

In the ERC-230 study, during long-term use (up to 52 weeks), prasterone was well tolerated [15], with $82 \%$ of the total of 530 women enrolled in the study completing the 52-week treatment. The most common adverse reaction was increased vaginal discharge (14\%), and 4 women discontinued treatment because of this adverse reaction. Abnormal Pap smear results with negative HPV status occurred in $1.6 \%$ of women treated with Intrarosa ${ }^{\circledR}$ for 52 weeks [3]. A single case of ovarian cancer was reported in the group of 1,196 women taking prasterone at a dose of $6.5 \mathrm{mg}$, which corresponds to an incidence rate which exceeds the value seen in the population of women of the same age, however the case was found before the start of treatment, and the patient had a BRCA1 mutation [3].

Both in the studies with a 12-week time horizon and in the 52-week study, the plasma levels of oestrogenic and androgenic DHEA metabolites in prasterone-treated patients were elevated in relation to the baseline, but were still within the normal range for postmenopausal women $[20,21]$. A pooled analysis of the results of steroid sex hormone determination performed using validated mass-spectrometry assays in patients treated with prasterone $(n=723)$ and in the placebo group ( $n=266$ ) (data from studies ERC-210, ERC-230, ERC-231 and ERC-238) showed that at week 12 the serum testosterone concentration in women taking prasterone was significantly higher compared to the baseline values and concentrations determined in the placebo group [21]. Oestradiol levels were also elevated in patients treated with prasterone, but the increase was significant compared to the baseline values and concentrations in the placebo group after excluding the subjects who had a serum oestrogen signature or a pattern of sex steroids typical of women having taken oestrogens [21]. The concentrations of oestradiol (only in women without oestrogen signatures) and testosterone remained statistically significantly higher than the baseline values after $\leq 52$ weeks of prasterone therapy in the ERC-230 study [20], but they remained within the normal range for serum steroid concentrations in postmenopausal women - oestradiol: < $10 \mathrm{pg} / \mathrm{ml}$; testosterone: $<260 \mathrm{pg} / \mathrm{ml}[20,21]$.

The assessment of endometrial safety of prasterone performed in a population of 668 women (with 389 receiving prasterone for a total of 52 weeks) showed no abnormal histological changes [22]. The absence of endometrial effect of intravaginal prasterone therapy is due to the intracrine mechanism of action of DHEA, and the lack of enzymes converting DHEA to oestrogens (mainly aromatase) in the endometrium. Consequently, intravaginal prasterone therapy does not increase the systemic exposure of the endometrium to proliferative hormonal factors.

A study investigating the level of patient acceptance of intravaginal administration of pessaries using an applicator was conducted in a group of women taking prasterone $(n=254)$ or placebo $(n=110)$. The administration of pessaries with the applicator was positively evaluated by $94.1 \%$ of women in the placebo group, and $91.7 \%$ women in the prasterone group. The instructions for using the applicator were considered as easy to understand by $100 \%$ women in the placebo group, and $98.8 \%$ women in the prasterone group. Almost all women reported that they had no difficulty 
using the applicator (95\% and $94.1 \%$ in the placebo and prasterone groups, respectively) [23].

\section{Conclusions}

- Intrarosa ${ }^{\circledR}$ is the first intravaginal drug containing prasterone. It is an innovative medicinal product indicated for the treatment of moderate to severe vulvar and vaginal atrophy in postmenopausal women. Intravaginal prasterone therapy involves the supply of both oestrogens and androgens to vaginal tissues.

- Clinical studies with a high level of evidence have shown that Intrarosa ${ }^{\circledR}$ significantly reduces symptoms of postmenopausal vaginal atrophy in women after the menopause, compared to placebo.

- Intrarosa ${ }^{\circledR}$ is both well tolerated and safe, and even long-term use of the drug does not elevate the serum levels of steroid sex hormones above the normal range for postmenopausal women.

\section{Disclosure}

The authors report no conflict of interest.

\section{References}

1. Management of symptomatic vulvovaginal atrophy: 2013 position statement of The North American Menopause Society. Menopause 2013; 20: 888-902.

2. Portman DJ, Gass MLS. Genitourinary syndrome of menopause: new terminology for vulvovaginal atrophy from the International Society for the Study of Women's Sexual Health and The North American Menopause Society. Menopause 2014; 21: 1-6.

3. Heo YA. Prasterone: A Review in Vulvovaginal Atrophy. Drugs Aging 2019; 36: 781-788.

4. Labrie F, Martel C, Pelletier G. Is vulvovaginal atrophy due to a lack of both estrogens and androgens? Menopause 2017; 24: 452-461.

5. Paszkowski T, Skrzypulec-Plinta V, Dębski R. Pomenopauzalna atrofia urogenitalna - nowe spojrzenie na stary problem. Menop Rev 2015; 14 : $1-4$

6. Traish AM, Vignozzi L, Simon JA, at al. Role of androgens in female genitourinary tissue structure and function: implications in the genitourinary syndrome of menopause. Sex Med Rev 2018; 6: 558-571.

7. Palacios S, Nappi RE, Bruyniks N, et al. The European Vulvovaginal Epidemiological Survey (EVES): prevalence, symptoms and impact of vulvovaginal atrophy of menopause. Climacteric 2018; 21: 286-291.

8. Shifren JL, Gass ML. The North American Menopause Society recommendations for clinical care of midlife women. Menopause 2014; 21 : 1038-1062.

9. Labrie F, Bélanger A, Pelletier G, et al. Science of intracrinology in postmenopausal women. Menopause 2017; 24: 702-712.

10. Intrarosa - charakterystyka produktu leczniczego.

11. Food and Drug Administration. Guidance for industry. Estrogen and estrogen/progestin drug products to treat vasomotor symptoms and vulvar and vaginal atrophy symptoms - recommendation for clinical evaluation, 2003.

12. Labrie F, Archer DF, Bouchard C, et al. Intravaginal dehydroepiandrosterone (prasterone), a highly efficient treatment of dyspareunia. Climacteric 2011; 14: 282-288.
13. Archer DF, Labrie F, MD, Bouchard C, at al. Treatment of pain at sexual activity (dyspareunia) with intravaginal dehydroepiandrosterone (prasterone). Menopause 2015; 22: 950-963.

14. Labrie F, Archer DF, Koltun W, at al. Efficacy of intravaginal dehydroepiandrosterone (DHEA) on moderate to severe dyspareunia and vaginal dryness, symptoms of vulvovaginal atrophy, and of the genitourinary syndrome of menopause. Menopause 2016; 23: 243-256.

15. Labrie F, Archer DF, Bouchard C, at al. Prasterone has parallel beneficial effects on the main symptoms of vulvovaginal atrophy: 52-week openlabel study. Maturitas 2015; 81: 46-56.

16. Labrie F, Archer DF, Martel C, et al. Combined data of intravaginal prasterone against vulvovaginal atrophy of menopause. Menopause 2017; 24: 1246-1256.

17. Labrie F, Derogatis L, Archer DF, et al. Effect of intravaginal prasterone on sexual dysfunction in postmenopausal women with vulvovaginal atrophy. J Sex Med 2015; 12: 2401-2412.

18. Bouchard C, Labrie F, Derogatis L, et al. Effect of intravaginal dehydroepiandrosterone (DHEA) on the female sexual function in postmenopausal women: ERC-230 open-label study. Horm Mol Biol Clin Investig 2016; 25: 181-190.

19. Labrie F, Montesino M, Archer DF, et al. Influence of treatment of vulvovaginal atrophy with intravaginal prasterone on the male partner. Climacteric 2015; 18: 817-825

20. Ke Y, Gonthier R, Jean-Nicolas Simard J-N, at al. Serum steroids remain within the same normal postmenopausal values during 12-month intravaginal 0.50\% DHEA. Horm Mol Biol Clin Invest 2015; 24: 117-129.

21. Martel C, Labrie F, Archerb DF, at al. Serum steroid concentrations remain within normal postmenopausal values in women receiving daily $6.5 \mathrm{mg}$ intravaginal prasterone for 12 weeks. J Steroid Biochem Mol Biol 2016; 159: 142-153.

22. Portman DJ, MD,Labrie F, MD, Archer DF, at al. Lack of effect of intravaginal dehydroepiandrosterone (DHEA, prasterone) on the endometrium in postmenopausal women. Menopause 2015; 22: 1289-1295.

23. Montesino M, Labrie F, Archer DF, at al. Evaluation of the acceptability of intravaginal prasterone ovule administration using an applicator. Gynecol Endocrinol, 2016; 32: 240-245. 